\title{
Cultivation and Serial Transfer of the Slime Mould, Dictyostelium discoideum in Liquid Nutrient Medium
}

\author{
By M. SUSSMAN \\ Brandeis University, Waltham, Massachusetts, U.S.A.
}

(Received 20 December 1960)

\begin{abstract}
SUMMARY
A method is described for the routine cultivation and serial passage of the slime mould Dictyostelium discoideum in liquid medium in association with Aerobacter aerogenes. The growth rates of several strains have been measured under these conditions.
\end{abstract}

\section{INTRODUCTION}

The cellular slime moulds grow by feeding upon bacteria. Although they can be maintained in axenic culture (Bradley \& Sussman, 1952), the difficulty of preparing the medium, the slow growth rate and the low yield of organism preclude the use of such cultures for ordinary purposes. In the past, the slime moulds have been cultivated exclusively on solid media. Investigators have thereby been faced, in performing many kinds of experiment, with serious technical difficulties which could have been avoided were it possible to cultivate these organisms in liquid media and to achieve reproducibly high growth rates and yields of organism. It was observed that Dictyostelium discoideum would grow luxuriantly in static liquid culture in association with a chromogenic pseudomonad of unknown species (Sussman, 1956a). However, the excessive slime production and attendant clumping by the bacteria made it difficult to obtain reproducible growth curves or to harvest the slime mould free from the bacterial associate. It was also observed that incubation of $D$. discoideum in a concentrated suspension of washed Aerobacter aerogenes in buffer permitted adequate growth and serial passage of the slime mould (Sussman, 1956a). Since then, a detailed procedure has been worked out by Gerisch (1960) under conditions similar to those just mentioned but with Escherichia coli as the associate bacterium at a concentration of $10^{10}$ bacteria $/ \mathrm{ml}$. A yield of $10^{7} \mathrm{myxamoebae} / \mathrm{ml} .(0 \cdot 8 \mathrm{mg}$. dry weight) and a doubling time of $3 \mathrm{hr}$. were achieved. Unfortunately, cultivation of myxamoebae with concentrated suspensions of pre-grown bacteria in buffer has certain basic defects. The task of harvesting and washing the bacteria, if it is to be a routine procedure, introduces a high chance of contamination with airborne bacteria and fungi. In practice, it has been impossible to maintain cultures uncontaminated for even a single passage, at least in our hands. Furthermore, the required concentration of bacteria makes it necessary that one grow, harvest, and wash about $7 \mathrm{l}$. bacterial culture to prepare $1 \mathrm{l}$. of slime mould culture. This precludes large-scale cultivation for biochemical experiments. The present communication describes a method which permits the routine cultivation and serial passage in liquid medium of $D$. discoideum in association with $\boldsymbol{A}$. aerogenes and which eliminates the disadvantages noted above. 
METHODS

Organisms. Two diploid strains of Dictyostelium discoideum (designated RA, H-1) and a haploid derivative (44-14) of strain RA were used. Stock cultures were started from single clone isolates and were maintained by mass plating on SM agar (Sussman, 1951 ; (g./l.): yeast extract, 1 ; Bactopeptone, 10 ; glucose, $10 ; \mathrm{K}_{2} \mathrm{HPO}_{4}, 1 \cdot 0 ; \mathrm{KH}_{2} \mathrm{PO}_{4}$, 1.5; $\mathrm{MgSO}_{4}, 0.5$; agar, 20) with Aerobacter aerogenes.

Composition of the liquid medium. Liquid medium A contained the following constituents (g./1.): Bacto-yeast extract, 0.5 ; Bactopeptone, 5 ; glucose, $5 ; \mathrm{KH}_{2} \mathrm{PO}_{4}, 2 \cdot 25$; $\mathrm{K}_{2} \mathrm{HPO}_{4} \cdot 12 \mathrm{H}_{2} \mathrm{O}, 1 \cdot 5 ; \mathrm{MgSO}_{4} \cdot 7 \mathrm{H}_{2} \mathrm{O}, 0.5$; to final $\mathrm{pH} 6-6 \cdot 3$. The medium was dispensed in $18 \mathrm{ml}$. lots into $125 \mathrm{ml}$. Erlenmeyer flasks (cotton-wool plugged) and sterilized by autoclaving at $121^{\circ}$.

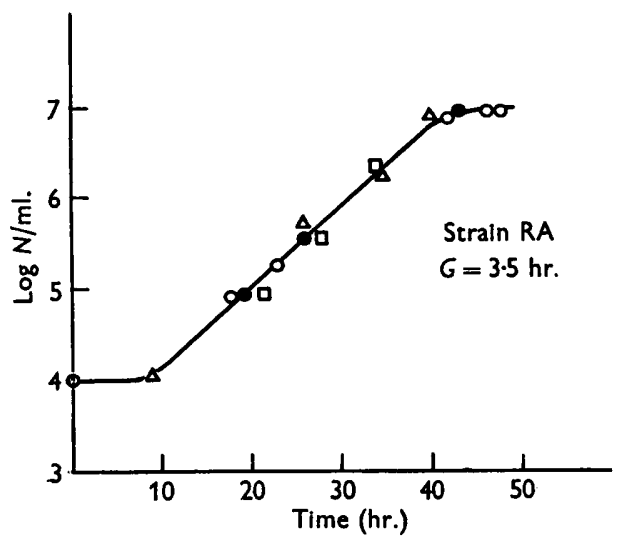

Fig. 1

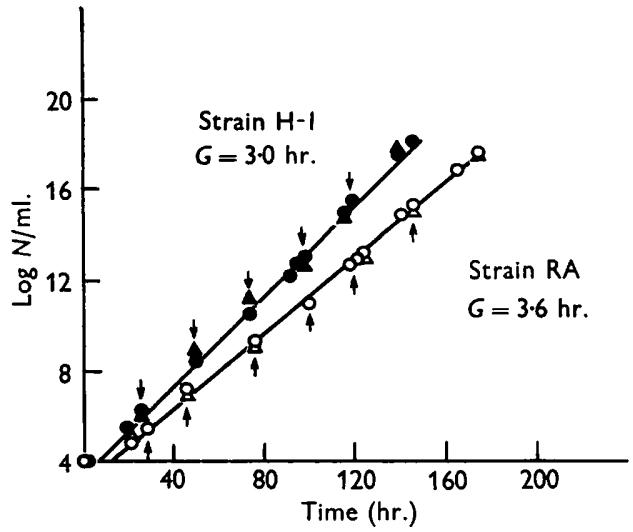

Fig. 2

Fig. 1. The culture cycle of Dictyostelium discoideum, strain RA, in medium A. Open and closed circles, squares and triangles represent independent experiments. $(N=$ total no. cells.)

Fig. 2. Sustained exponential growth of Dictyostelium discoideum strains RA and H-1 by serial subculture in medium A. Each culture started at a population density of $10^{4}$ myxamoebae $/ \mathrm{ml}$. and was allowed to reach 1 to $3 \times 10^{6} \mathrm{myxamoebae} / \mathrm{ml}$. before subculture. The arrows designate the times at which the subcultures were made. The ordinate is a cumulative count of organisms, i.e. the last point for one subculture was the zero time point for the next. Circles represent total counts; triangles represent viable counts.

Method of cultivation. One ml. of myxamoeboid or spore suspension at 1 or $2 \times 10^{5}$ organisms $/ \mathrm{ml}$. and $1 \mathrm{ml}$. of a $48 \mathrm{hr}$. broth (Sussman, 1951) culture of Aerobacter aerogenes served as the inocula. The cultures were incubated at $22^{\circ}$ on a reciprocating shaker (200 cyc./min.; 1.5 in. stroke).

Growth measurement. Direct counts were made in quadruplicate or octuplicate with a Levy haemocytometer. Viable counts were made from clonal platings (Sussman, 1951) in quintuplicate.

\section{RESULTS}

Figure 1 summarizes the data from four separate culture cycles with Dictyostelium discoideum strain RA. The inoculum of strain RA consisted of spores taken from 7-day growth plates. (The terminal fruiting stage was reached at 3 days under these 
conditions.) A lag phase of about $10 \mathrm{hr}$. was encountered in the liquid medium. As might be expected, the duration of the lag was found to depend on the age of the inoculum. Spores from older plates displayed a longer lag period, those from younger plates a shorter one, and inoculation of logarithmic phase myxamoebae eliminated it completely. A characteristic exponential phase encompassed about 10 divisions. This indicates a constant generation time and a degree of viability exceeding $95 \%$, a figure verified by the viable count data shown in Fig. 2. The logarithmic phase generation time, $(t \log 2) /\left(\log N / N_{0}\right)$, was $3.5 \mathrm{hr}$. In a similar series strain 44-14 (the haploid derivative of RA) yielded a generation time of $3.0 \mathrm{hr}$., comparable to the values obtained by growth on solid media (Sussman, 1956b). The stationary phase is seen in Fig. 1 to have begun at a population density of $8 \times 10^{6}$ myxamoebae $/ \mathrm{ml}$., a yield equivalent to that obtained by Gerisch (1960) with pre-grown washed Escherichia coli in buffer.

Figure 2 shows the growth of strains RA and H-1 during 7 and 6 serial passages, respectively, in medium $A$, when inoculated during the logarithmic phase of growth. Exponential growth was maintained over the course of about $\mathbf{4 0}$ divisions, representing a $2^{40}$ dilution of the original protoplasm. The total and viable counts agreed within the limits of random sampling error $( \pm 20 \%$ c.v. $)$. No signs of contamination by airborne bacteria or fungi were seen. In agreement with previous findings (Gerisch, 1960), myxamoebae taken from the liquid culture and dispensed on solid substrata aggregated and fruited normally. The generation time for strain RA was calculated to be $3 \cdot 6 \mathrm{hr}$. and for strain $\mathrm{H}-1,3 \cdot 0 \mathrm{hr}$., a significant difference.

Dictyostelium discoideum can be cultivated in considerably larger volumes of medium $\mathbf{A}$ than used in the above experiments. An inoculum of $10^{4}$ myxamoebae $/ \mathrm{ml}$. in 1 l. medium A contained in a Fernbach flask and aerated through a sintered glass sparger attained a generation time of 3-4 $\mathrm{hr}$. and a yield of $10^{7} \mathrm{myxamoebae} / \mathrm{ml}$.

\section{DISCUSSION}

The fact that cellular slime moulds can be grown in the laboratory in liquid media and without special treatment of the bacterial associate raises an important ecological question. In the past, great emphasis has been placed upon the soil habitat in evaluating the ecological position of the Acrasiales (Singh, 1946; Raper, 1951). The conditions for laboratory cultivation in broth are clearly not so critical that they could not be reproduced in nature. A systematic examination of streams and pond water habitats particularly during periods of bacterial bloom might well reveal a new dimension to the natural history of these organisms and might turn up new varieties.

This work was supported by Grant G-12900 from the National Science Foundation and Grant C-4057 from the National Institutes of Health. The excellent technical assistance of Miss Linda King in the performance of this work is gratefully acknowledged.

\section{REFERENCES}

Bradley, S. G. \& Sussman, M. (1952). Growth of amoeboid slime moulds in one membered culture. Arch. Biochem. Biophys. 39, 462.

Gerisch, G. (1960). Ein Submerskulturverfahren für Entwicklungsphysiologische Untersuchungen an Dictyostelium discoideum. Naturwissenschaften, 47, 656. 
RAPER, K. B. (1951). Isolation, cultivation, and conservation of simple slime moulds. Quart. Rev. Biol. 26, 169.

Singr, B. N. (1946). Soil Acrasiae and their bacterial food supply. Nature, Lond. 157, 133.

Sussman, M. (1951). Origin of cellular heterogeneity in the slime moulds, Dictyosteliaceae. J. exp. Zool. 118, 407.

Sussman, M. (1956a). Biology of the cellular slime moulds. Annu. Rev. Microbiol. 10, 21. Sussman, M. $(1956 b)$. On the relation between growth and morphogenesis in Dictyostelium discoideum. Biol. Bull., Woods Hole, 110, 91. 